Quim. Nova, Vol. 34, No. 6, 992-995, 2011

\title{
PYRROLIZIDINE ALKALOIDS OF Senecio sp FROM PERU
}

\section{Liliana Ruiz Vásquez and Matías Reina Artiles*}

Instituto de Productos Naturales y Agrobiología, CSIC, Avda. Astrofísico Francisco Sánchez, 3, 38206, La Laguna, Tenerife, Spain Azucena González Coloma

Instituto de Ciencias Agrarias-(ICA), CSIC, Serrano 115-dpdo, 28006 Madrid, Spain

Raimundo Cabrera Pérez

Unidad de Fitopatología, Facultad de Biología, Universidad de La Laguna (ULL), Avda. Astrofísico Francisco Sánchez, s/n, 38204, La Laguna, Tenerife, Spain

Lastenia Ruiz Mesia

Laboratorio de Investigación en Productos Naturales Antiparasitarios de la Amazonía, Universidad Nacional de la Amazonía Peruana (LIPNAA-UNAP), AA. HH. "Nuevo San Lorenzo" Pasaje los Paujiles S/N - San Juan, Iquitos, Peru

Recebido em 20/9/10; aceito em 15/12/10; publicado na web em 14/3/11

\begin{abstract}
Six pyrrolizidine alkaloids (PAs) (two saturated macrocyclic, three unsaturated macrocyclic and one unsaturated seco-macrocyclic) were isolated from native Peruvian Senecio species. The structures of these alkaloids were established by a complete NMR spectroscopic analysis, chemical transformations and comparison of their NMR data with those published for similar alkaloids. Three PAs were then tested for antifungal activity against Fusarium moniliforme, F. (Sheldon), F. oxysporum fs. lycopersici (Scheldt) and F. solani (Mart), no significant activity being observed.
\end{abstract}

Keywords: Senecio; pyrrolizidine alkaloids; 19-acetoxysenkirkine.

\section{INTRODUCTION}

Pyrrolizidine alkaloids (PAs) are widely distributed in numerous plant families such as Asteraceae, Boraginaceae, Fabaceae, Convolvulaceae, Orchidaceae, Poaceae and Lamiaceae. ${ }^{1}$ However, pyrrolizidine alkaloids are found predominantly in the genera Senecio (Asteraceae), Crotalaria (Fabaceae) and Heliotropium (Boraginaceae). In Peru, the genus Senecio comprises about 230 species, most of which are endemic and with restricted geographic distribution, the habitat of these species lying between 500 and $4700 \mathrm{~m}$ above sea level. ${ }^{2}$ It should be noted that species of Senecio are used as popular remedies in folk medicine in different forms of preparation such as infusions, decoctions and poultices (to treat kidney disorders and ulcers), while there is great concern regarding public health since these species contain PAs with hepatotoxic activity. ${ }^{3}$ It is therefore necessary to identify the PA-containing plants to limit their use, or at least establish the maximum tolerated dose. Pyrrolizidine alkaloids (PAs) are of great interest as biopesticides. ${ }^{4}$ This has prompted us to carry out a phytochemical study of the genus Senecio, in addition to studying its biological activity.

Here, we wish to report on the isolation and characterization of six alkaloids of four species of Senecio from Peru: Senecio lasiocephalus $(\mathbf{1}, \mathbf{2})$, S. usgorensis (3), S. chiquianensis (4) and S. laricifolius (5, 6). Alkaloid 3 was previously isolated from Senecio megaphyllus (Costa Rica) and Senecio usgorensis (Peru), and compound 6 from S. laricifolius (Peru). ${ }^{5}$

*e-mail: mreina@ipna.csic.es

\section{EXPERIMENTAL}

\section{General experimental procedures}

1D and $2 \mathrm{D}{ }^{1} \mathrm{H},{ }^{13} \mathrm{C}$ NMR spectra were measured on a Bruker AMX $500 \mathrm{MHz}$ spectrometer with pulsed-field gradient in $\mathrm{CDCl}_{3}$ (500 MHz for ${ }^{1} \mathrm{H}$ and $125 \mathrm{MHz}$ for ${ }^{13} \mathrm{C}$ ), using this solvent as internal standard. Optical rotations were measured at room temperature on a Perkin Elmer 343 Plus polarimeter. IR spectra were measured on a Perkin Elmer 1600 spectrophotometer. EIMS results were recorded on a VG-Micromass ZAB-2F instrument at $70 \mathrm{eV}$. Alumina (Merck Art. 1076-1077) was used for column chromatography, TLC and preparative TLC. Alkaloids were visualized with Dragendorff's reagent.

\section{Plant material}

All plants were collected in May 2008, at Markahuamachuco (Department of La Libertad), Peru, in the natural habitats between 3542-3597 $\mathrm{m}$ above sea level. Voucher specimens have been deposited at the Herbarium Amazonense of the Universidad Nacional de la Amazonía Peruana (Iquitos-Loreto): S. laricifolius H.B.K (voucher number 10456), S. usgorensis Cuart (voucher number 10453), S. chiquianensis Cabrera (voucher number 10455) and S. lasiocephalus (voucher number 10454).

\section{Extraction and isolation of Pas}

The dried samples were extensively extracted with ethanol at room temperature over $72 \mathrm{~h}$ and the extracts evaporated to dryness under reduced pressure. The crude $\mathrm{EtOH}$ extract was treated with a mixture of $\mathrm{H}_{2} \mathrm{SO}_{4} 0.5 \mathrm{M}$ and $\mathrm{CH}_{2} \mathrm{Cl}_{2}(1: 1)$. The aqueous phase was reduced with zinc dust for 4-6 h, filtered, basified with $30 \% \mathrm{NH}_{4} \mathrm{OH}$ 
$(\mathrm{pH}=9)$ and the alkaloids were extracted with $\mathrm{CH}_{2} \mathrm{Cl}_{2}$. The purification was performed by column chromatography on neutral alumina using an EtOAc-MeOH gradient solvent system and subsequent purification with PTLC $(20 \times 20 \mathrm{~cm}, 0.25 \mathrm{~mm})$ to obtain platyphylline and platyphylline $\mathrm{N}$-oxide (1, 2, S. lasiocephalus), 13,19-epoxysenecionine (3, S. usgorensis), senecivernine (4, S. chiquianensis), senecionine and 19-acetoxysenkirkine $(\mathbf{5}, \mathbf{6}$, S. laricifolius $)$.

\section{Platyphylline (1)}

Mp 96.5-98.7 ${ }^{\circ} \mathrm{C}$ (hexane-EtOAc): $[\alpha]_{\mathrm{D}}^{25^{\circ}}-46.0^{\circ}\left(\mathrm{c}, 0.094, \mathrm{CHCl}_{3}\right)$ [lit. $[\alpha]_{\mathrm{D}}^{25^{\circ}}-46^{\circ}$ in $\left.\mathrm{CHCl}_{3}\right]^{6}$; EIMS m/z (int. rel., \%): 337 (7) $[\mathrm{M}]^{+}, 226$ (5), 222 (4), 212 (6), 211 (50), 180 (4), 141 (10), 140 (100), 138 (57), 123 (53), 122 (93), 96 (22), 82 (50), 80 (11) and 55 (16). HREIMS $\mathrm{m} / \mathrm{z}[\mathrm{M}]^{+} 337.1887$ (16), calculated for $\mathrm{C}_{18} \mathrm{H}_{27} \mathrm{NO}_{5} .{ }^{1} \mathrm{H}$ and ${ }^{13} \mathrm{C} \mathrm{NMR}$ data identical with those reported. ${ }^{7,8}$

\section{Platyphylline N-oxide (2)}

Amorphous solid: $[\alpha]_{\mathrm{D}}^{25^{\circ}}-38.2^{\circ}\left(\mathrm{c}, 0.136, \mathrm{CHCl}_{3}\right)$. EIMS $\mathrm{m} / z$ (int. rel., \%): 353 (2), 338 (3), 337 (11), 336 (6), 335 (7), 226 (11), 211 (51), 153 (20), 140 (97), 139 (14), 138 (84), 122 (66), 119 (74), 96 (35), 82 (100), 80 (22), and 55 (30). ESI-MS m/z [M+1] 354.1917 (2), calculated for $\mathrm{C}_{18} \mathrm{H}_{28} \mathrm{NO}_{6}$

\section{3,19-epoxysenecionine (3)}

EIMS m/z (int. rel., \%): 349 (25) [M]+, 304 (6), 288 (7), 262 (4), 218 (8), 138 (29), 136 (100), 120 (91), 119 (63), 95 (27), 94 (46), 93 (59), 84 (23), 80 (27), 67 (10), 53 (19); HREIMS m/z [M]+ 349.1530, calculated for $\mathrm{C}_{18} \mathrm{H}_{23} \mathrm{NO}_{6} .{ }^{1} \mathrm{H}$ and ${ }^{13} \mathrm{C}$ NMR data identical with those reported. ${ }^{9}$

\section{Senecivernine (4)}

Amorphous solid: $[\alpha]_{\mathrm{D}}^{25^{\circ}}-14.9^{\circ}\left(\mathrm{c}, 0.35, \mathrm{CHCl}_{3}\right)$, [lit. $[\alpha]_{\mathrm{D}}^{25^{\circ}}-34.9^{\circ}$ $(\mathrm{EtOH})] \cdot{ }^{10} \mathrm{IR}\left(\mathrm{CHCl}_{3}\right) v_{\text {máx }} 3400,1718,1710,1654,1560,1508$, 1458, 1255, 1160, $764 \mathrm{~cm}^{-1}$. EIMS $m / z$ [M] 335 (6), 291 (10), 248 (12), 218 (2), 153 (53), 136 (84), 120 (100), 93 (68), 81 (54), 55 (46); HREIMS, $m / z[\mathrm{M}]^{+} 335.0020$, calculated for $\mathrm{C}_{18} \mathrm{H}_{25} \mathrm{NO}_{6} \cdot{ }^{1} \mathrm{H}$ and ${ }^{13} \mathrm{C}$ NMR data identical with those reported. ${ }^{10}$

\section{Senecionine (5)}

Amorphous solid: $[\alpha]_{\mathrm{D}}^{25^{\circ}}-67.2^{\circ}\left(c, 0.054, \mathrm{CHCl}_{3}\right)\left[\right.$ lit. $[\alpha]_{\mathrm{D}}^{25^{\circ}}-56.0^{\circ}$ in $\left.\mathrm{CHCl}_{3}\right]^{6}$. EIMS m/z [M] 335 (1), 333 (6), 291 (3), 248 (2), 220 (2), 153 (5), 136 (100), 120 (98), 119 (70), 93 (72), 80 (35), 53 (27); HREIMS, $m / z[\mathrm{M}]^{+} 335.1783$, calculated for $\mathrm{C}_{18} \mathrm{H}_{25} \mathrm{NO}_{5} .{ }^{1} \mathrm{H}$ and ${ }^{13} \mathrm{C}$ NMR data identical with those reported. ${ }^{7,8}$

\section{9-acetoxysenkirkine (6)}

Amorphous solid: IR $\left(\mathrm{CHCl}_{3}\right) v_{\text {máx }} 3400,1720,1650$ and 1612 $\mathrm{cm}^{-1}$, ESI (Electro Spray Ionization) $\mathrm{m} / z$ : $[\mathrm{M}+1]^{+} 424.1964$ (74), calculated for $\mathrm{C}_{21} \mathrm{H}_{30} \mathrm{NO}_{8}$. Data for ${ }^{1} \mathrm{H}$ and ${ }^{13} \mathrm{C}$ NMR are given in Table 2.

\section{Reduction of 2}

Platyphylline $\mathrm{N}$-oxide $\left(85.6 \mathrm{mg}\right.$ ) was treated with $\mathrm{H}_{2} \mathrm{SO}_{4} 0.5 \mathrm{~N}$ and zinc dust with stirring for $5 \mathrm{~h}$ at room temperature. The reaction product was filtered, basified with $\mathrm{NH}_{4} \mathrm{OH} 30 \%$ to $\mathrm{pH}=8-9$ and extracted with $\mathrm{CH}_{2} \mathrm{Cl}_{2}$ several times. After removal of the solvent platyphylline $(\mathbf{1}, 57.0 \mathrm{mg}, 70.0 \%)$ was obtained.

\section{Antifungal assays of PAs}

The antifungal activity of platyphylline, platyphylline $\mathrm{N}$-oxide and 19-epoxysenecionine was tested against the following species of phytopathogenic fungi: Fusarium moniliforme (Sheldon), F. oxysporum fs. lycopersici (Scheldt) and F. solani (Mart) (Spanish Type Culture Collection (CECT) codes, CECT 2152, CECT 2715, and CECT 2199, respectively) and estimated as mycelial growth inhibition. The products were dissolved in ethanol and incorporated in PDA culture media ( $2 \%$ final concentration of solvent). The dose series used was: 0.01, 0.1 and $1.0 \mathrm{mg} / \mathrm{mL}$. For each experiment 8 replicates, inoculated with the appropriate fungal sp., were incubated at $25^{\circ} \mathrm{C}$ and the colony diameters measured after $48 \mathrm{~h}$. Control experiments were ethanol. The inhibition values were determined with the SPS program.

\section{RESULTS AND DISCUSSION}

Alkaloid extract yield ranged between 0.03 and $0.1 \%$ with respect to plant dry weight. Senecio lasiocephalus and S. usgorensis showed the highest alkaloid content. Two saturated macrocyclic diester PAs, platyphylline and its $\mathrm{N}$-oxide $(\mathbf{1 , 2})$, three unsaturated macrocyclic diester PAs, 13,19-epoxysenecionine (3), senecivernine (4) senecionine (5), and one unsaturated macrocyclic diester seco-PAs, 19-acetoxysenkirkine (6), have been isolated from their respective alkaloid extracts (Table 1). All structures were established by spectroscopic data, chemical transformations and correlations of these data with those reported for similar alkaloids (Figure 1).
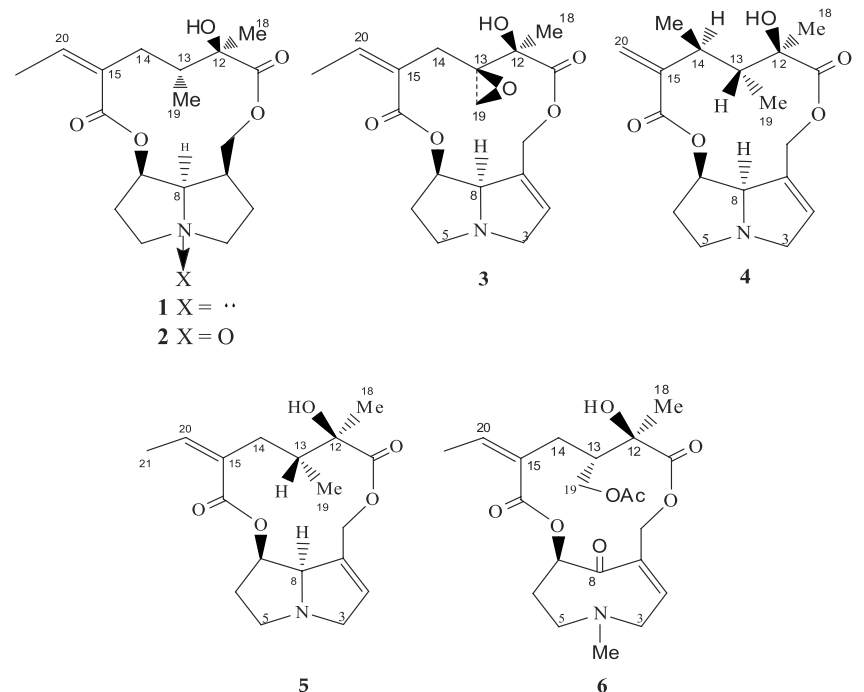

Figure 1. Chemicals structures of alkaloids 1-6

Table 1. Weight of plant material, alkaloid extract and pure compounds isolated

\begin{tabular}{|c|c|c|c|c|c|c|}
\hline Plant & $\begin{array}{l}\text { Weight of the ground } \\
\text { floor }\end{array}$ & Ethanolic extract & Alkaloid extract & Compounds isolated & Weight (mg) & Yield $\%$ \\
\hline \multirow[t]{2}{*}{ S. lasiocephalus } & $654.3 \mathrm{~g}$ & $149.6 \mathrm{~g}$ & $690.4 \mathrm{mg}$ & 1 & 178.3 & $2.7 \times 10^{-2}$ \\
\hline & & & & 2 & 484.7 & $7.4 \times 10^{-2}$ \\
\hline S. usgorensis & $811.4 \mathrm{~g}$ & $138.2 \mathrm{~g}$ & $203.0 \mathrm{mg}$ & 3 & 203.0 & $2.5 \times 10^{-2}$ \\
\hline S. chiquianensis & $801.7 \mathrm{~g}$ & $165.3 \mathrm{~g}$ & $290.3 \mathrm{mg}$ & 4 & 17.1 & $2.1 \times 10^{-3}$ \\
\hline \multirow[t]{2}{*}{ S. laricifolius } & $537.1 \mathrm{~g}$ & $185.5 \mathrm{~g}$ & $176.8 \mathrm{mg}$ & 5 & 10.1 & $1.9 \times 10^{-3}$ \\
\hline & & & & 6 & 29 & $5.4 \times 10^{-3}$ \\
\hline
\end{tabular}


Alkaloids $\mathbf{1}$ and $\mathbf{2}$ were identified as platyphylline and its $\mathrm{N}$ oxide $\left({ }^{1} \mathrm{H}\right.$ and $\left.{ }^{13} \mathrm{C} \mathrm{NMR}\right)$, previously isolated from different species of Senecio, ${ }^{11,12}$ and were related via chemical by reduction of 2 with $\mathrm{H}_{2} \mathrm{SO}_{4} / \mathrm{Zn}$ dust. The most noteworthy differences observed in the ${ }^{1} \mathrm{H}$ and ${ }^{13} \mathrm{C}$ NMR spectra of both compounds were the largest deshielding of $\mathrm{H}-8$ at $\delta_{\mathrm{H}} 4.00(1 \mathrm{H}, \mathrm{dd}, J=8.2$ and $6.1 \mathrm{~Hz})$ and resonance of carbons $\delta_{\mathrm{C}} 70.4(\mathrm{t}, \mathrm{C}-3), 68.3(\mathrm{t}, \mathrm{C}-5)$ and $86.0(\mathrm{~d}, \mathrm{C}-8)$ with respect to alkaloid $\mathbf{1}$, suggesting the presence of an extra oxygen molecule in the form of $\mathrm{N}$-oxide. ${ }^{13}$

The structure of alkaloids 3-5 were elucidated by mono- and bidimensional NMR and by comparison with previously reported spectroscopic data. ${ }^{8-10}$ Alkaloid 6, proved to be otonecine esterified with 5-ethylidene-2-hydroxy-2-methyl-3-methylacetyl-hexanodioic acid and was identified with 19-acetoxysenkirkine based on the following points: the IR spectrum showed absorption bands at $v_{\max }$ $3400,1720,1650$ and $1612 \mathrm{~cm}^{-1}$ which could be attributed to a carbonyl group, an ester, double bonds and a trans-annular carbonyl group $\mathrm{O}=\mathrm{C} \cdots \mathrm{N}-\mathrm{Me},{ }^{14}$ respectively. Its mass spectrum presents a molecular ion at $m / z, 424.1964(74)[\mathrm{M}+1]^{+}$along with fragments of a macrocyclic ester, necic acid and otonecine base, characteristic of secopyrrolizidine alkaloids. ${ }^{15,16}$ The ${ }^{1} \mathrm{H}$ NMR and ${ }^{13} \mathrm{C}$ spectra of alkaloid 6 were very similar to those of 12 -acetylsenkirkine, an alkaloid previously isolated from S. phillipicus and S. illinitus ${ }^{17}$ except for the presence of a $\mathrm{CH}_{2} \mathrm{OCOCH}_{3}$ group at $\mathrm{C}-13$ at $\delta_{\mathrm{H}} 4.11(1 \mathrm{H}, \mathrm{dd}, J=$ 12.2 y $2.9 \mathrm{~Hz}, \mathrm{H}-19 \mathrm{a}), 4.21$ (1H, dd, $J=6.1,11.8 \mathrm{~Hz}, \mathrm{H}-19 \mathrm{~b}), \delta_{\mathrm{C}} 61.9$ (t, C-19), two signals connected with carbon resonances of $\delta_{\mathrm{C}} 33.7(\mathrm{t}$, C-14), 42.8 (d, C-13), 76.1 (s, C-12) and 170.9 (s, $\mathrm{OCOCH}_{3}$ ) by an HMBC experiment. The complete NMR data of 19-acetoxysenkirkine (6) are presented in Table 2. The relative stereochemistry of alkaloids 3 and $\mathbf{6}$ was determined by a NOESY experiment, and observed NOE effects are shown in Figure 2.

Three APs, platyphylline, platyphylline $\mathrm{N}$-oxide and 13,19-epoxysenecionine, were tested against the following fungal species $\mathrm{Fu}$ sarium moniliforme (Sheldon), F. oxysporum fs. lycopersici (Scheldt)

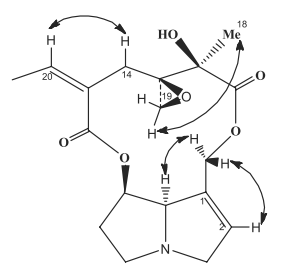

3

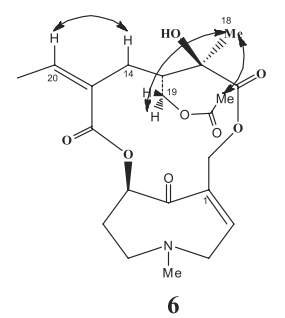

Figure 2. NOESY of 13,19-epoxysenecionine (3) and 19-acetoxysenkirkine (6)

Table 2. ${ }^{1} \mathrm{H},{ }^{13} \mathrm{C}, \mathrm{COSY}$, HSQC and HMBC NMR data of 6

\begin{tabular}{|c|c|c|c|c|}
\hline \multirow[t]{2}{*}{ Proton } & \multirow{2}{*}{$\delta\left(\mathrm{J}_{\mathrm{H}-\mathrm{H}}\right.$ en $\left.\mathrm{Hz}\right)$} & \multirow[t]{2}{*}{ COSY } & \multicolumn{2}{|c|}{ Correlated carbons } \\
\hline & & & HSQC & ${ }^{\mathrm{a}} \mathrm{H} \mathrm{HMBC}$ \\
\hline 1 & - & & $134.2 \mathrm{~s}$ & - \\
\hline 2 & $6.2 \mathrm{~s}$ & $\mathrm{H}-3 \mathrm{a}, \mathrm{H}-3 \mathrm{~b}$ & $136.9 \mathrm{~d}$ & $\mathrm{C}-3, \mathrm{C}-9$ \\
\hline $3 \alpha$ & 3.46 br d (18.5) & $\mathrm{H}-2, \mathrm{H}-3 \mathrm{~b}$ & $59.3 \mathrm{t}$ & $\mathrm{N}-\mathrm{Me}, \mathrm{C}-5$ \\
\hline $3 \beta$ & 3.33 br d (18.5) & $\mathrm{H}-2, \mathrm{H}-3 \mathrm{a}$ & & N-Me, C-5 \\
\hline $5 \alpha$ & $2.94 \mathrm{~m}$ & H-5b, H-6a, H-6b & $53.9 \mathrm{t}$ & N-Me, C-7 \\
\hline $5 \beta$ & $2.79 \operatorname{td}(12.4,4.0)$ & H-5a, H-6a, H-6b & & $\mathrm{N}-\mathrm{Me}, \mathrm{C}-3, \mathrm{C}-6, \mathrm{C}-7$ \\
\hline $6 \alpha$ & $2.58 \mathrm{~m}$ & H-5a, H-5b, H-6b & $35.8 \mathrm{t}$ & C-5 \\
\hline $6 \beta$ & $2.36 \mathrm{dq}(3.9,11.4)$ & H-5a, H-5b, H-6a & & C-5 \\
\hline $7 \alpha$ & $5.05 \mathrm{t}(2.0)$ & H-6a, H-6b & $72.2 \mathrm{~d}$ & $\mathrm{C}-5, \mathrm{C}-16$ \\
\hline 8 & - & - & $191.0 \mathrm{~s}$ & - \\
\hline $9 \mathrm{~d}$ & $5.42 \mathrm{~d}(11.2)$ & $\mathrm{H}-9 \mathrm{u}$ & $64.2 \mathrm{t}$ & $\mathrm{C}-1, \mathrm{C}-2, \mathrm{C}-11$ \\
\hline $9 \mathrm{u}$ & $4.40 \mathrm{~d}(11.2)$ & $\mathrm{H}-9 \mathrm{~d}$ & & $\mathrm{C}-1, \mathrm{C}-2, \mathrm{C}-11$ \\
\hline 11 & - & - & $177.1 \mathrm{~s}$ & - \\
\hline 12 & - & - & $76.1 \mathrm{~s}$ & - \\
\hline 13 & $2.05 \mathrm{~m}$ & H-19a, H-19b, H-14a, H-14b & $42.8 \mathrm{~d}$ & - \\
\hline $14 \mathrm{a}$ & $2.02 \mathrm{~m}$ & $\mathrm{H}-13, \mathrm{H}-14 \mathrm{~b}$ & $33.7 \mathrm{t}$ & $\mathrm{C}-12, \mathrm{C}-13, \mathrm{C}-14$ \\
\hline $14 \mathrm{~b}$ & $2.21 \mathrm{~m}$ & $\mathrm{H}-13, \mathrm{H}-14 \mathrm{a}$ & & C-12, C-13, C-14 \\
\hline 15 & - & - & $130.7 \mathrm{~s}$ & - \\
\hline 16 & - & - & $166.0 \mathrm{~s}$ & - \\
\hline 18 & $1.42 \mathrm{~s}$ & - & $25.0 \mathrm{q}$ & $\mathrm{C}-12, \mathrm{C}-13$ \\
\hline $19 \mathrm{a}$ & $4.11 \mathrm{dd}(12.2,2.9)$ & $\mathrm{H}-19 \mathrm{~b}$ & $61.9 \mathrm{t}$ & C-12, C-13, C-14 \\
\hline $19 b$ & $4.21 \mathrm{dd}(11.8,6.1)$ & $\mathrm{H}-19 \mathrm{a}$ & & C-12, C-13, C-14 \\
\hline 20 & $5.92 \mathrm{q}(7.2)$ & $\mathrm{H}-21$ & $138.3 \mathrm{~d}$ & $\mathrm{C}-21, \mathrm{C}-14$ \\
\hline 21 & $1.90 \mathrm{dd}(7.1,1.4)$ & $\mathrm{H}-20$ & $15.4 \mathrm{q}$ & $\mathrm{C}-14, \mathrm{C}-15$ \\
\hline $\mathrm{OCOCH} \underline{3}_{3}$ & $2.04 \mathrm{~s}$ & - & $21.0 \mathrm{q}$ & C-19 \\
\hline $\mathrm{OCOCH}_{3}$ & - & - & $170.9 \mathrm{~s}$ & - \\
\hline $\mathrm{N}-\mathrm{Me}$ & $2.18 \mathrm{~s}$ & - & $41.1 \mathrm{q}$ & $\mathrm{C}-3, \mathrm{C}-5$ \\
\hline
\end{tabular}

${ }^{\mathrm{a}}$ Multiplicities of ${ }^{13} \mathrm{C}$ NMR were established by DEPT experiment 
and $F$. solani (Mart) as previously described, ${ }^{18}$ but no significant antifungal activity was observed.

\section{SUPPLEMENTARY MATERIAL}

Available on http://quimicanova.sbq.org.br, in PFD file, with free access. ${ }^{1} \mathrm{H}$ NMR spectra of alkaloids 1-4 and 6.

\section{ACKNOWLEDGEMENTS}

This work was supported by a Collaborative Research Grant from CSIC-CONCYTEC, National University of the Peruvian Amazonia, Iquitos, Peru, Ministerio de Educación y Ciencia, Spain (CTQ200914629-C02-01) and a predoctoral MAEC-AECID fellowship to L. R. Vásquez.

\section{REFERENCES}

1. Silva, C. M.; Bolzan, A. A.; Heinzmann, B.; Quim. Nova 2006, 29, 1047.

2. Vision, T. J.; Dillon, M.; Arnaldoa 1996, 4, 23.

3. Bolzan, A. A.; Silva, C. M.; Francescato, L. N.; Murari, A. L.; Silva, N. S.; Heldwein, C. G.; Heinzmann, B.; Lat. Am. J. Pharm. 2007, 26, 619; Angeles, C. T.; Fukusaki, D. I.; Díaz, P. M.; Anales Científicos UNALM, Cadima, H. V., ed.; Editorial Agraria: Lima, 2001, nº 47, 37; Hammond, G. B.; Fernandez, I. D.; Villegas, L. F.; Vaisberg, A. J.; J. Ethnopharmacol. 1998, 61, 17; Rojas, R.; Bustamante, B.; Bauer, J.; Fernandez, I.; Alban, J.; J. Ethnopharmacol. 2003, 88, 199; De Feo, V.; J. Ethnopharmacol. 2003, 85, 243; Mattocks, A. R.; Chemistry and Toxicology of Pyrrolizidine Alkaloids, Academic Press: London, 1986, chap. 7.
4. Bull, L. B.; Culvenor, C. C. J.; Dick, A. T.; The pyrrolizidine alkaloids. Their chemistry, pathogenicity and other biological properties, NorthHolland Publ.: Amsterdam, 1968, p. 249; Domínguez, D. M.; Reina, M.; Santos-Guerra, A.; Santana, O.; Agullo, T.; López-Balboa, C.; GonzálezColoma, A.; Biochem. Syst. Ecol. 2008, 36, 153.

5. Bohlmann, F.; Zdero, C.; Jakupovic, J.; Grenz, M.; Castro, V.; King, R. M.; Robinson, H.; Vincent, P. D.; Phytochemistry 1986, 25, 1151.

6. Rizk, A.-F. M., ed.; The pyrrolizidine alkaloids; plant sources and properties, CRC Press: Boca Raton, 1990, p. 40.

7. Logie, C. G.; Grue, M. R.; Liddell, J. R.; Phytochemistry 1994, 37, 43.

8. Roeder, E.; Phytochemistry 1990, 29, 11.

9. Hartmann, T.; Witte, L.; Ehmke, A.; Theuring, C.; Rowell-Rahier, M.; Pasteels, J. M.; Phytochemistry 1997, 45, 489.

10. Röeder, E.; Wiendenfel, F.; Pastewka, H.; Planta Med. 1979, 37, 131.

11. Paiva, J. A.; Soares-Barata, L. E.; Trigo, J. R.; Biochem. Syst. Ecol. 2004, 32, 1219.

12. Perez-Castorena, A. L.; Arciniegas, A.; Martinez, F.; Villaseñor, J. L.; Romo de Vivar, A.; Biochem. Syst. Ecol. 2000, 28, 279

13. Barrero, A.; Álvarez, R.; An. Quim. 1991, 87, 386; Karchesy, J. J.; Deinzer, M. L.; Griffin, D. A.; J. Agric. Food Chem. 1984, 32, 1057.

14. Briggs, L. H.; Cambie, R. C.; Candy, B. J.; O`Donovan, G. M.; Russell, R. H.; Seelye, R. N.; J. Chem. Soc. 1965, 2409.

15. Wasim, A.; Abdul, O. K.; Abdul, M.; Ergum, F.; Bilge, S.; J. Nat. Prod. 1992, 55, 1764.

16. Asada Y.; Furuya, T.; Chem. Pharm. Bull. 1984, 32, 475.

17. González, A. G.; de la Fuente, G.; Reina, M.; Loyola, L. A.; Planta Med. 1986, 52, 160

18. Macías, F. A.; Simonet, A. M.; D’Abrosca, B.; Maya, C. C.; Reina, M.; González-Coloma, A.; Cabrera, R.; Giménez, C.; Villarroel, L.; J. Chem. Ecol. 2009, 35, 35. 


\section{PYRROLIZIDINE ALKALOIDS OF Senecio sp FROM PERU}

\section{Liliana Ruiz Vásquez and Matías Reina Artiles*}

Instituto de Productos Naturales y Agrobiología, CSIC, Avda. Astrofísico Francisco Sánchez, 3, 38206, La Laguna, Tenerife, Spain Azucena González Coloma

Instituto de Ciencias Agrarias-(ICA), CSIC, Serrano 115-dpdo, 28006 Madrid, Spain

Raimundo Cabrera Pérez

Unidad de Fitopatología, Facultad de Biología, Universidad de La Laguna (ULL), Avda. Astrofísico Francisco Sánchez, s/n, 38204 , La Laguna, Tenerife, Spain

\section{Lastenia Ruiz Mesia}

Laboratorio de Investigación en Productos Naturales Antiparasitarios de la Amazonía, Universidad Nacional de la Amazonía Peruana (LIPNAA-UNAP), AA. HH. "Nuevo San Lorenzo" Pasaje los Paujiles S/N - San Juan, Iquitos, Peru

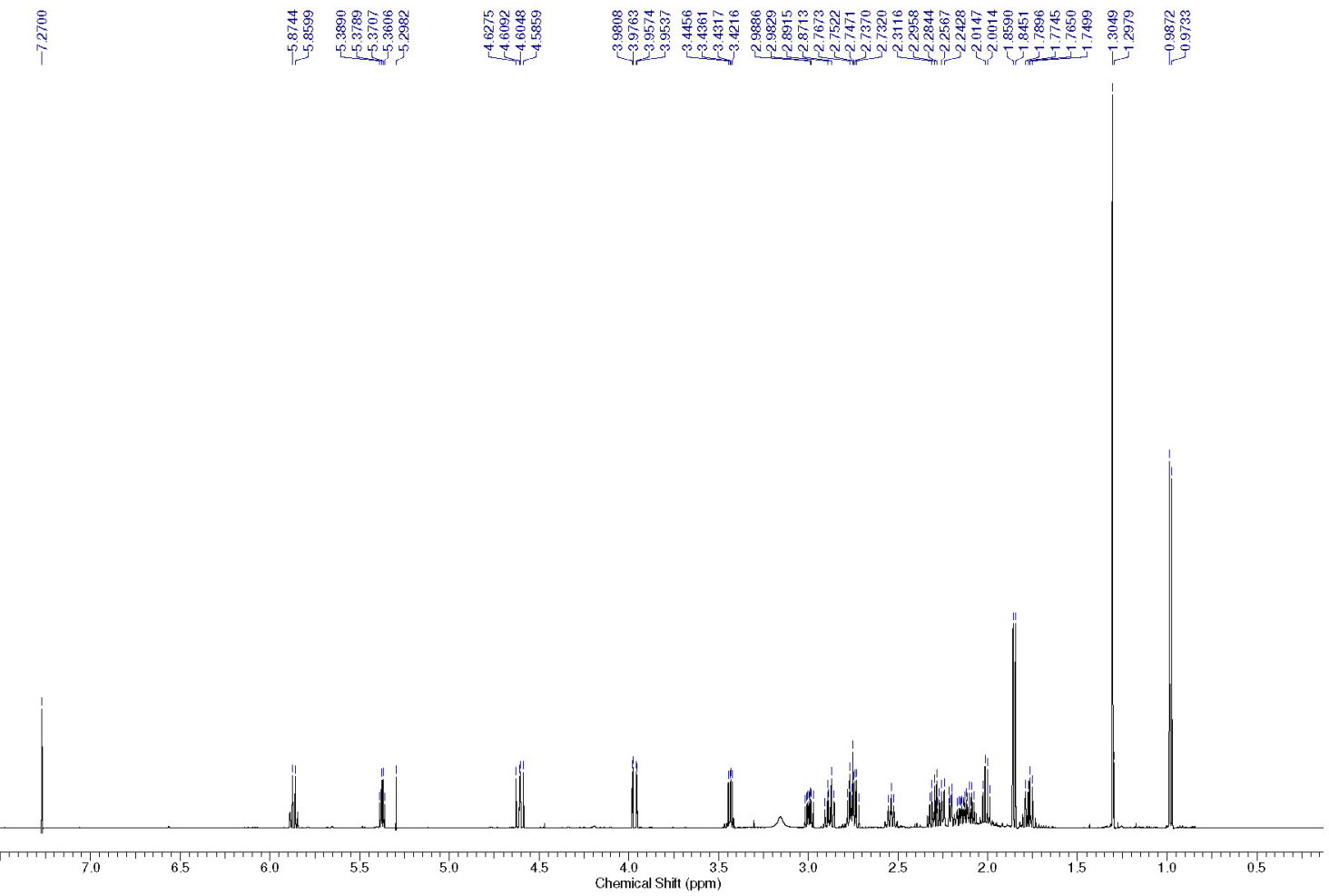

Figure 1S. ${ }^{1} H$ NMR spectrum of 1 


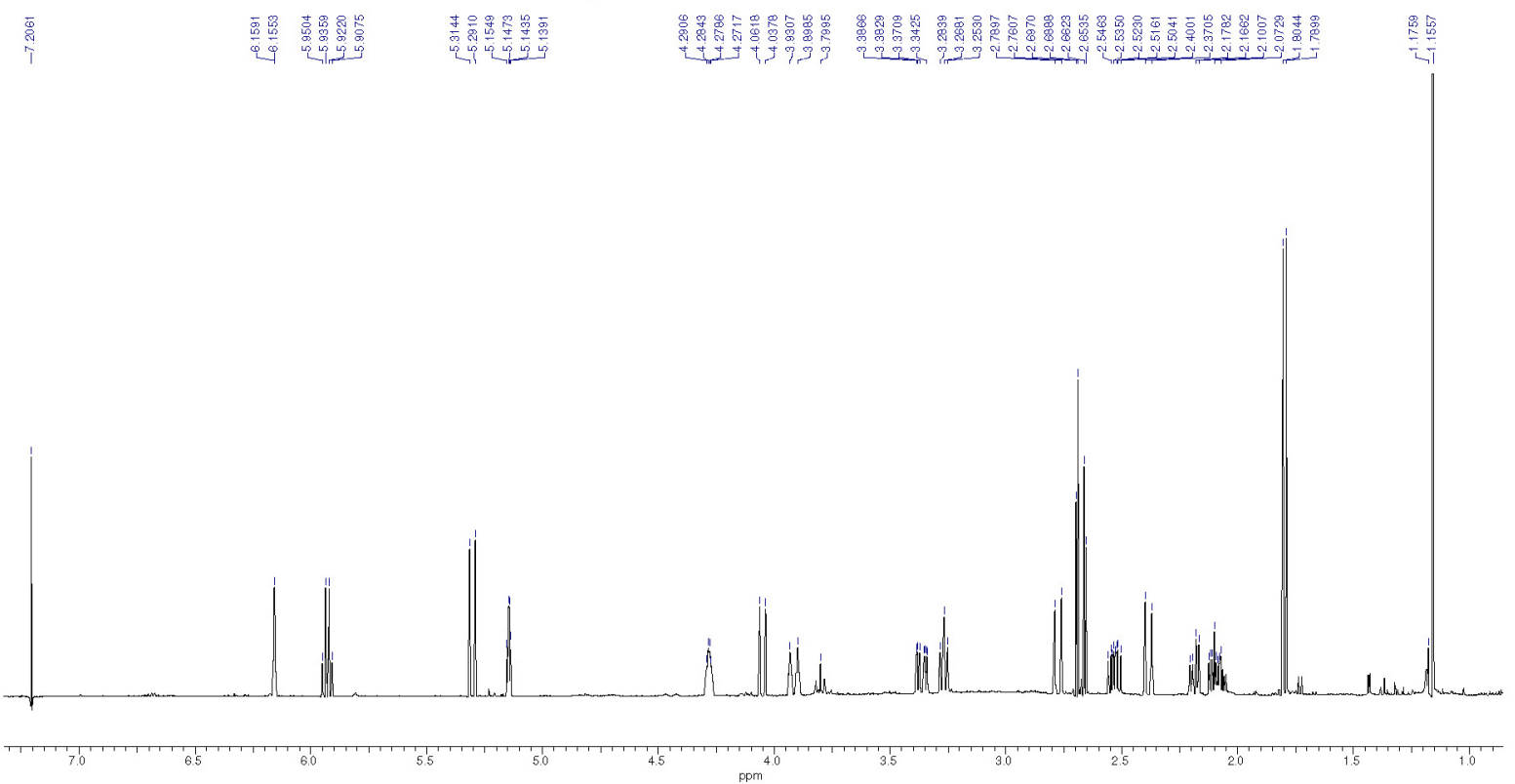

Figure $2 S .{ }^{1} H$ NMR spectrum of 2

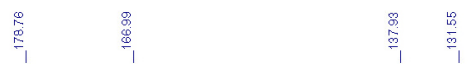

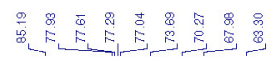

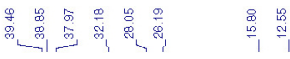

. 


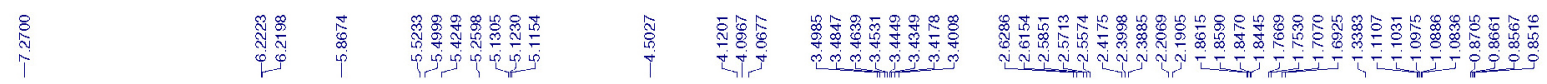

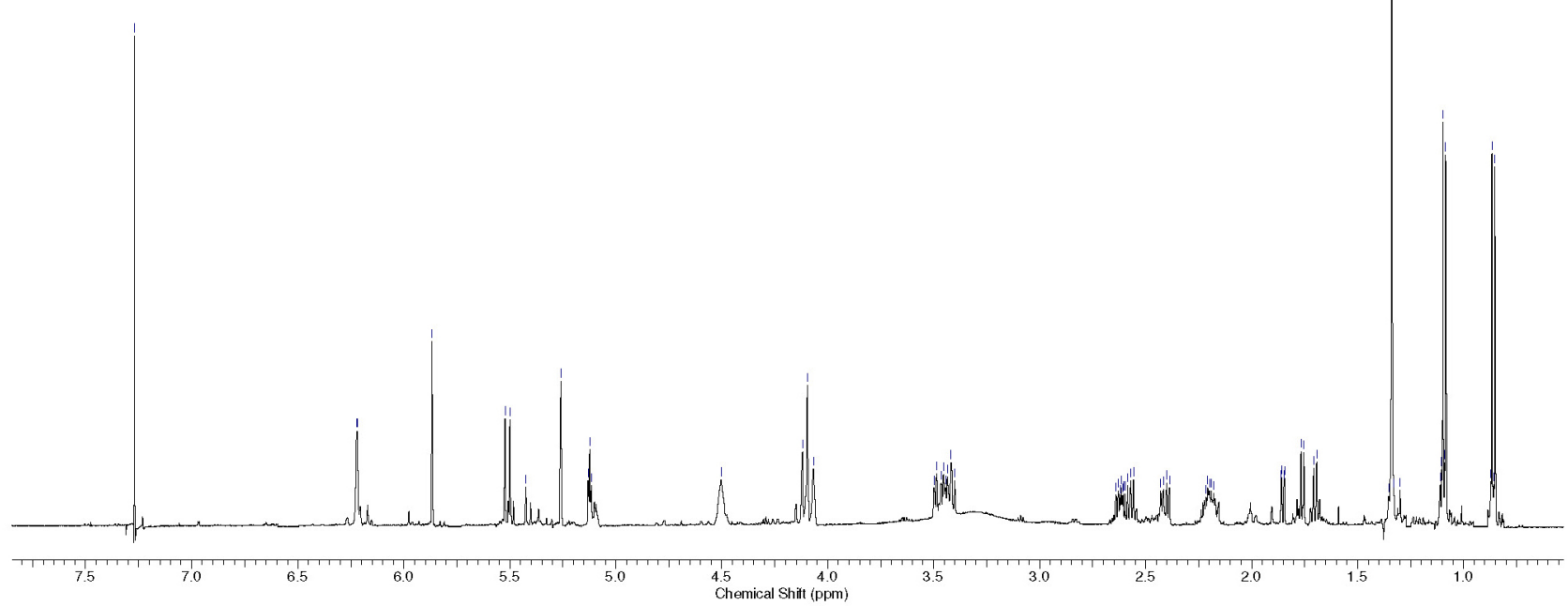

Figure $4 S .{ }^{1} H$ NMR spectrum of 3

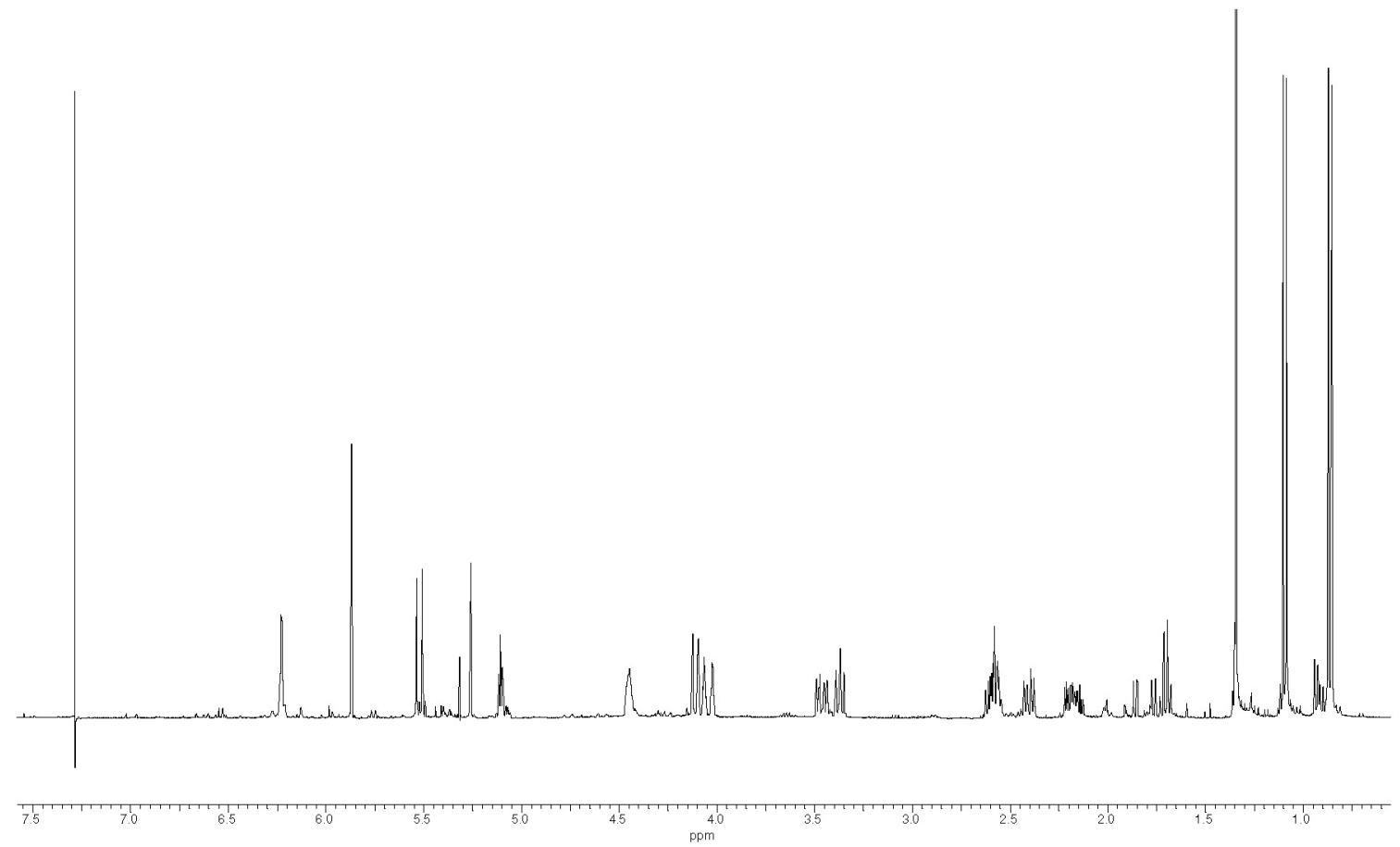

Figure 5S. ${ }^{1} H$ NMR spectrum of 4 


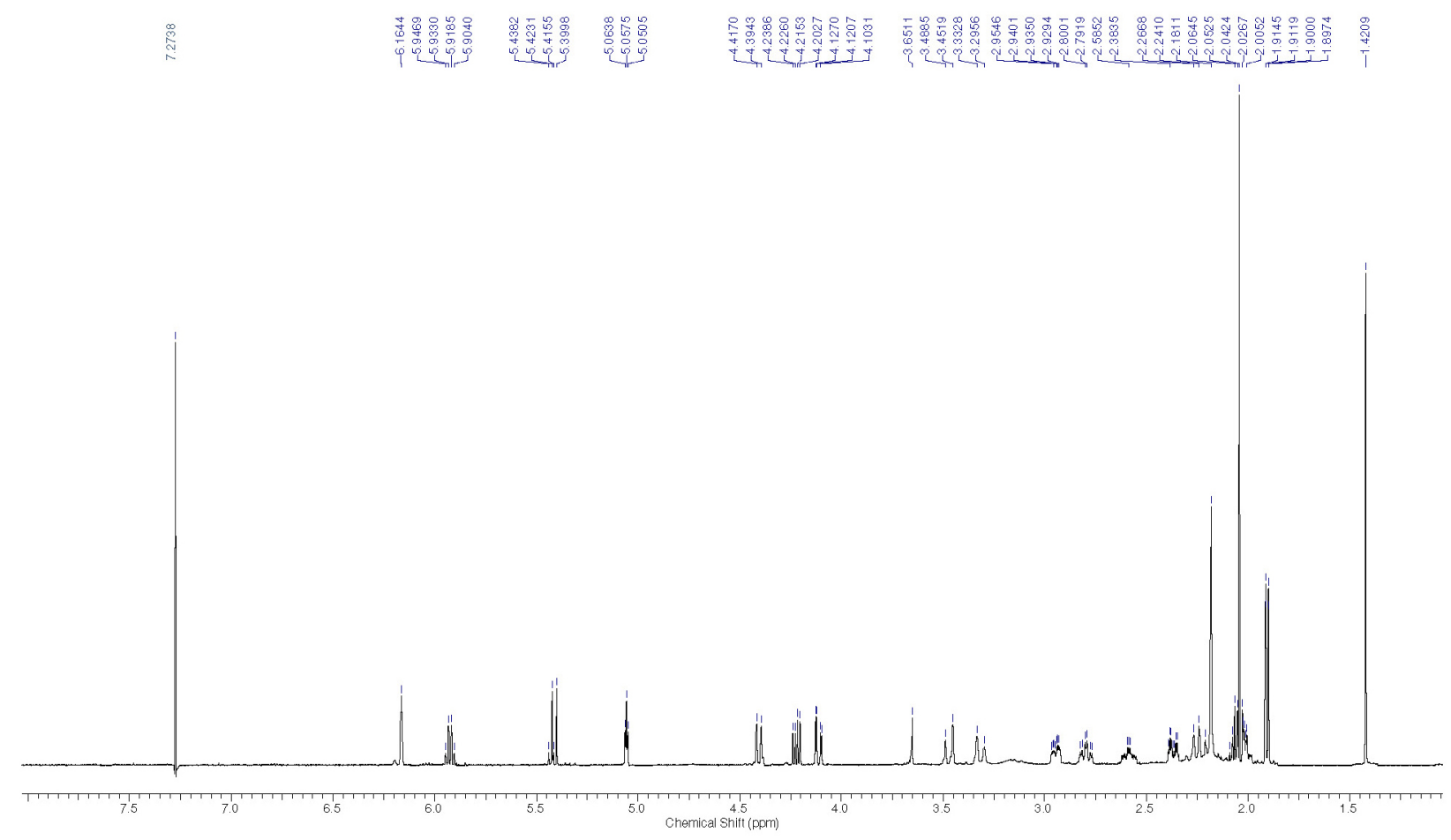

Figure 6S. ${ }^{1} H$ NMR spectrum of 6 